\title{
Comportamiento bi-modal de una fundición gris y un acero estructural al carbono en corrosión atmosférica marina acelerada
}

\author{
Bi-modal behavior of gray cast iron and carbon \\ structural steel in accelerated marine \\ atmospheric corrosion
}

Alfredo Artigas ${ }^{1}$, Rodrigo Seco ${ }^{1}$, Alberto Monsalve ${ }^{1}$,
Konstantin Sipos ${ }^{1}$, Nelson Garza-Montes-de-Oca ${ }^{2}$

\begin{abstract}
${ }^{1}$ Departamento de Ingeniería Metalúrgica, Facultad de Ingeniería, Universidad de Santiago de Chile, Av. L. B. O’Higgins 3363, Estación Central, Santiago, Chile. CP 9160000. e-mail: alfredo.artigas@usach.cl, rodrigo.seco@usach.cl, alberto.monsalve@usach.cl, ksiposh@yahoo.com

${ }^{2}$ Facultad de Ingeniería Mecánica y Eléctrica, Universidad Autónoma de Nuevo León, San Nicolás de los Garza, Nuevo León, México C.P 66450. email: nelson.garza@gmail.com
\end{abstract}

\section{RESUMEN}

De acuerdo a datos del último censo de la producción de la industria de las fundiciones [1], se observó que aproximadamente el 46\% de la producción en la industria de las aleaciones ferrosas corresponde a la fundición gris. Por ello que se hace interesante el investigar la resistencia que posee este material a la corrosión atmosférica, y así determinar el real tiempo de vida útil a la intemperie. Los del comportamiento de las fundiciones grises bajo corrosión atmosférica son escasos, debido estos toman largos periodos de tiempo para la caracterización en tiempo real.

Con el propósito de validar un ensayo de corrosión atmosférica acelerada, se ha sometido a una fundición gris [2] y a un acero al carbono [3]. a 40 días de un ensayo acelerado [4], que involucra ciclos de humectación en cámara de niebla salina, lavado y secado, determinando el espesor corroído en cada tiempo. Estos resultados, luego fueron comparados, con los obtenidos por Southwell et al. [5] luego de 16 años de exposición a la intemperie en un ambiente marino (Canal de Panamá). El análisis de resultados reveló que ambos materiales presentan un comportamiento bi-modal, en concordancia con lo descrito por Melchers et al. [6, 7, 8], éste comportamiento se caracteriza por un punto de inflexión en la curva cinética de espesor corroído, lo que permite la validación de la metodología propuesta como medio para evaluar la resistencia a la corrosión atmosférica marina en poco tiempo.

Palabras clave: corrosión atmosférica, hierro, pérdida en peso, corrosión bacteriana.

\begin{abstract}
According to data gathered in the last Metal Foundry Industrial Production Survey [1], approximately 46\% of the industrial production of ferrous alloys is gray-iron. In this context the study of the atmospheric corrosion resistance of gray-iron becomes interesting to asset its useful life when subject to weathering conditions. The studies of gray-iron atmospheric corrosion behavior are scarce due to the fact that it takes long periods of time for its real-time characterization.

In order to validate an accelerated atmospheric corrosion test, a gray cast iron [2] and a carbon steel [3] were subjected to a 40 days accelerated testing, that includes wetting in a saline fog chamber, washing and drying [4], measuring the corroded thickness at each exposure time. These results were then compared with those obtained by Southwell et al [5] after 16 years of exposure in a marine environment (Panamá Canal). The result analysis reveals that both materials show a bimodal behavior, in accordance with those results reported by Melchers et al [6, 7, 8 ]. This behavior is characterized by an inflection point in the kinetic curve of corrosion, meanly related to the presence of bacteria in the interphase metal-corrosion products. The fact that the kinetic curve of corroded thickness shows an inflection point, allows the validation of the proposed methodology as a path to evaluate the atmospheric corrosion resistance in a short time test.
\end{abstract}


Keywords: atmospheric corrosion, iron, weight loss, bacterial corrosion.

\section{INTRODUCCIÓN}

En la actualidad existen pocos estudios sobre la resistencia a la corrosión atmosférica de los materiales en general y en particular de las aleaciones ferrosas. Algunos de ellos se han concentrado en caracterizar ambientes de corrosión [9] y otros en evaluar la resistencia a la corrosión atmosférica marina en aceros [10, 11] y fundiciones $[5,12,13]$. La realización de trabajos como estos tiene una desventaja en común y es que para su realización se requiere de años, por ello algunos autores y en particular este equipo de trabajo, se han concentrado en buscar la forma de reducir estos tiempos a través de la realización de experiencias de corrosión acelerada [4, 14-22], que tras poco tiempo y en laboratorio (días) puedan generar resultados concordantes con los obtenidos en años de exposición a la intemperie.

Continuando con la búsqueda de validar una metodología para la reducción de tiempos de investigación de la resistencia a la corrosión atmosférica marina de los materiales, es que este trabajo tiene por objetivo obtener resultados de espesor corroído en el tiempo para una fundición gris A48 [2] y un acero estructural al carbono A36 [3], para luego comparar estos resultados de laboratorio, obtenidos mediante un ensayo de corrosión acelerada [4], con los obtenidos tras años de exposición a la intemperie [5].

\subsection{Modelos de corrosión}

Como primera aproximación a la determinación de corrosión en el largo plazo, se han utilizado modelos del tipo potencial, como el presentado en la Ecuación 1, donde C representa el espesor corroído, t el tiempo de exposición y A y B son constantes. La forma de esta expresión surge del supuesto que la corrosión comienza inmediatamente y se produce de manera indefinida [7]. No obstante, existen ciertos productos de corrosión que protegen los materiales frente a este fenómeno y por tanto la concentración de oxígeno sobre el sustrato es distinta en el tiempo.

Con el propósito de considerar los elementos anteriormente descritos y que quedan excluidos en la determinación de la Ecuación 1, es que Melchers [23] propone que la corrosión en aleaciones ferrosas, es un fenómeno que cambia en el tiempo, identificándose dos fases; la primera, en que la corrosión es aeróbica y la segunda, anaeróbica influenciada principalmente por la actividad de bacterias reductoras de sulfato. Se encuentra así una relación entre espesor corroído y tiempo de exposición a la intemperie, del tipo bi-modal, como la ilustrada en la Figura 1.

$$
C=A t^{B}
$$

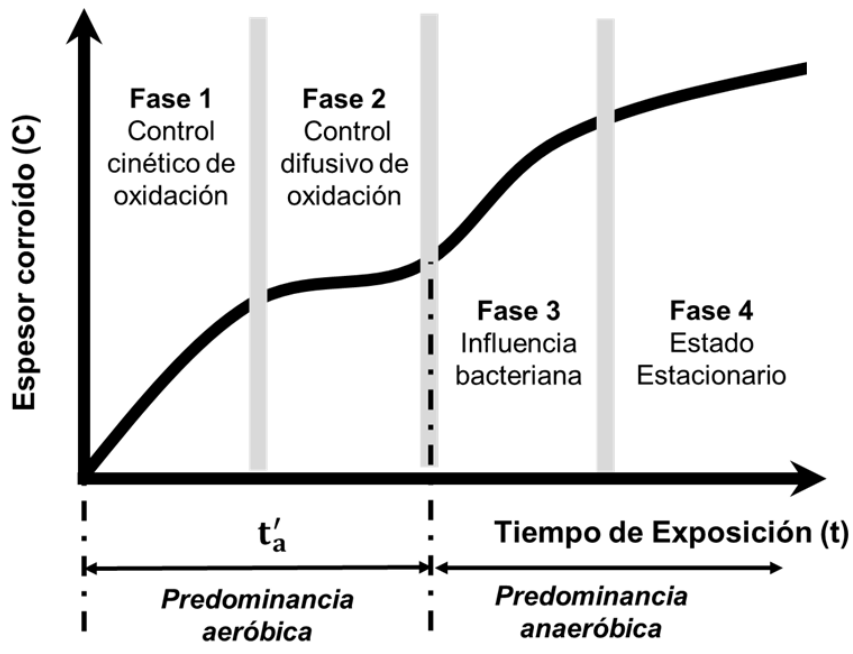

Figura 1: Esquema del modelo bi-modal para espesor corroído, mostrando sus principales fases.

El término t’ marca la transición de una predominancia a otra y de acuerdo a observaciones de datos experimentales [6] se alcanza durante los primeros años de exposición a la intemperie (3 a 6 años), por ello durante a la realización de ensayos de corrosión acelerada se pondrá énfasis en los primeros ciclos. 


\section{MATERIALES Y MÉTODOS}

A continuación, se presentarán las tres partes en que se dividen las experiencias y metodología utilizada.

\subsection{Ciclos de corrosión acelerada}

Para desarrollar esta etapa, se prepararon cupones de 50 x 30 x 6, todas en mm, de la fundición gris (FG) y acero estructural (AE). Estos cupones una vez medidos y pesados fueron incorporados a los ciclos de corrosión acelerada de 24 as de duración cada uno. Cada ciclo consta de las siguientes etapas, con un total de 24 (h) por ciclo:

1. 3 (h) en cámara de niebla salina, según la norma ASTM B117 [24].

2. 2 (h) de secado

3. 17 (h) en cámara de niebla salina

4. Lavado de las muestras con una ducha

5. 2 (h) de secado

La etapa de lavado, cumple un rol fundamental en la experiencia, ya que por un lado simula la erosión ambiental que experimenta el acero y por otro lado baja de manera considerable las concentraciones de $\mathrm{NaCl}$, lo cual permite la formación de la pátina protectora.

Las muestras estuvieron sometidas durante 40 ciclos (960 (h)), extrayendo muestras en los ciclos 3,5 , $6,9,10,12,15,20,25,30,35$ y 40 (se ha puesto el foco en los primeros ciclos, de acuerdo a lo comentario con anterioridad).

\subsection{Análisis morfológico de productos de corrosión}

Con la finalidad de evaluar la morfología, la compactación y grietas de los productos de corrosión presentes en los cupones, se realizó microscopía electrónica de barrido (SEM). Ya en un trabajo anterior del grupo de investigación [4], se demostró que empleando el ensayo de corrosión acelerada descrito en la sección 2.1, se pueden obtener en pocas semanas los productos de corrosión reportados en la literatura tras varios años de exposición a la intemperie [25], los resultados de este estudio [4], se identificaron, cuantificaron los productos de corrosión (Goethita, Lepidocrocita; Akaganeita, Hematita, Maghemita/Magnetita) que son concordantes con la corrosión a largo plazo, expresado en la literatura. Si bien es cierto los productos de corrosión formados en la fundición gris no fueron analizados, es esperable que presente este mismo tipo de oxidróxidos, tal como dice la literatura. [26, 27], no siendo relevantes para este estudio, pues lo que se desea replicar es el comportamiento bimodal del material frente a la corrosión atmosférica marina.

Para mantener adheridos al cupón los productos de corrosión, se pegó a este un film de aluminio con resina epóxica. Posteriormente se realizó un corte transversal con un disco de corte refrigerado. Una vez cortado el cupón recubierto fue montado en baquelita conductora para microscopía electrónica. Luego, los cupones montados fueron lijados, pulidos y luego atacados químicamente empleando una solución de ácido clorhídrico al 33\% durante 5 (s). Se realizó este procedimiento para muestras obtenidas tras el ciclo 30.

\subsection{Determinación de espesores corroídos}

El espesor corroído promedio se calculó a partir de la pérdida de peso (método gravimétrico) y las velocidades de corrosión mediante la Ecuación 2, tal como lo especifica la norma ASTM G1 [28].

$$
V=\frac{K W}{D A T}
$$

V es la velocidad de corrosión, $K$ es una constante que depende de las unidades finales de la velocidad de corrosión y de las unidades usadas en el resto de los parámetros, $\mathrm{W}$ es la pérdida de peso, D es la densidad de la muestra, A es el área total y $\mathrm{T}$ el tiempo de exposición. Se emplearon duplicados en cada uno de los ciclos estudiados.

\section{RESULTADOS Y DISCUSIÓN}

\subsection{Caracterización de materiales}

Se obtuvieron propiedades mecánicas y composiciones químicas de los materiales de estudio, estos resultados se presentan en las Tablas 1 y 2. Además, se muestran como referencias las exigidas por ASTM A48 y 
ASTM A36, como fundición gris (FG) y acero estructural respectivamente (AE). Adicionalmente en la Tabla 2 se presentan las composiciones químicas de las muestras sometidas a corrosión atmosférica marina por Southwell et al. [4]. La Figura 3 presenta las imágenes metalográficas para ambos materiales.

Tabla 1: Propiedades mecánicas.

\begin{tabular}{c|c|c|c|c} 
Muestra ID & $\begin{array}{c}\text { Tensión de } \\
\text { Fluencia } \\
\mathbf{0 , 2 \%} \begin{array}{c}\text { Deformación } \\
\text { (MPa) }\end{array}\end{array}$ & $\begin{array}{c}\text { Tensión } \\
\text { Máxima } \\
\mathbf{( M P a )}\end{array}$ & $\begin{array}{c}\text { Alargamiento } \\
\text { Sobre } \\
\mathbf{5 0 ~} \mathbf{~ m m} \\
\mathbf{( \% )}\end{array}$ & $\begin{array}{c}\text { Dureza } \\
\mathbf{( H B )}\end{array}$ \\
\hline Fundición gris & - & 152 & 1 & 151 \\
\hline REF ASTM A48 Gr. 20 & - & $140-152$ & - & $130-180$ \\
\hline Acero estructural & 351 & 476 & 34 & - \\
\hline REF ASTM A36 & mín. 250 & $400-550$ & mín. 21 & -
\end{tabular}

Tabla 2: Composiciones químicas.

\begin{tabular}{c|c|c|c|c|c|c|c|c}
$\mathbf{w} \%$ & $\mathbf{C}$ & $\mathbf{M n}$ & $\mathbf{P}$ & $\mathbf{S}$ & $\mathbf{S i}$ & $\mathbf{C r}$ & $\mathbf{N i}$ & $\mathbf{C u}$ \\
\hline $\begin{array}{c}\text { Fundición gris } \\
\text { (FG) }\end{array}$ & 3,61 & 0,89 & 0,18 & 0,014 & 2,75 & 0,026 & - & 0,035 \\
\hline Ref. ASTM A48 & $3,2-3,6$ & $0,5-0,9$ & 0,2 máx. & 0,2 máx. & $2,3-2,5$ & - & - & - \\
\hline FG Southwell [5] & 3,180 & 0,800 & 0,162 & 0,103 & 1,980 & 0,570 & 0,310 & 0,080 \\
\hline $\begin{array}{c}\text { Acero Estructural } \\
\text { (AE) }\end{array}$ & 0,157 & 0,7 & 0,011 & 0,026 & 0,181 & 0,042 & 0,054 & 0,085 \\
\hline Ref. ASTM A36 & 0,25 máx. & - & 0,03 máx. & 0,03 máx. & 0,04 máx. & - & - & - \\
\hline AE Southwell [5] & 0,240 & 0,480 & 0,040 & 0,027 & 0,008 & 0,030 & 0,051 & 0,080
\end{tabular}

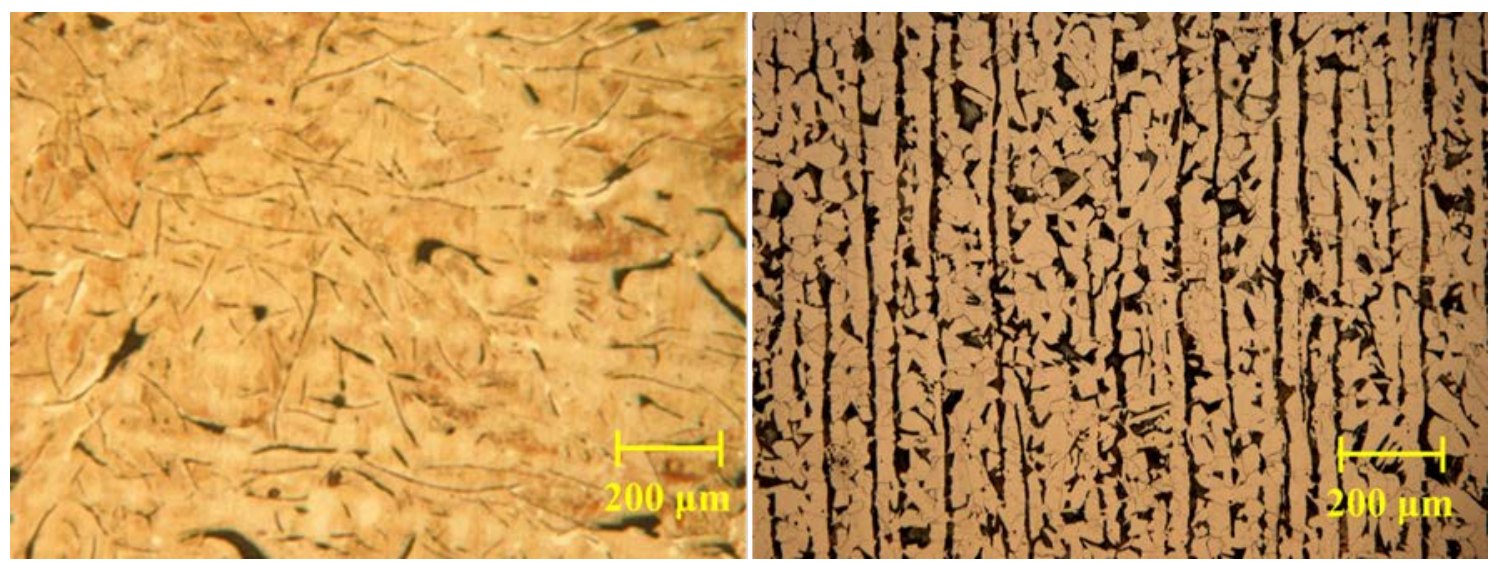

Figura 2: Metalografías de muestras de estudio; a la derecha la fundición gris y a la izquierda el acero estructural.

De acuerdo a los resultados anteriores, los materiales empleados cumplen con los requerimientos químicos y mecánicos explicitados en las normas de referencia. Además de acuerdo a sus imágenes metalográficas presentan estructuras típicas para este tipo de materiales, siendo una distribución homogénea de grafito laminar en una matriz ferrítico-perlítica para el caso de la fundición gris, y una dispersión de colonias perlíticas en los límites de granos equiaxiales de la matriz ferrítica, para el caso del acero estructural. 


\subsection{Análisis Morfológico de productos de corrosión}

Los resultados de microscopía electrónica para los materiales estudiados se presentan en las Figuras 3 y 4 , para fundición gris y acero estructural respectivamente. En ambos casos el sustrato corresponde a la porción clara en la zona inferior de las imágenes. Para el caso de la fundición gris, además se muestra un mapeo de carbono obtenido mediante la técnica EDS-SEM, en rojo se observan las regiones ricas en este elemento, como por ejemplo las láminas de grafito.

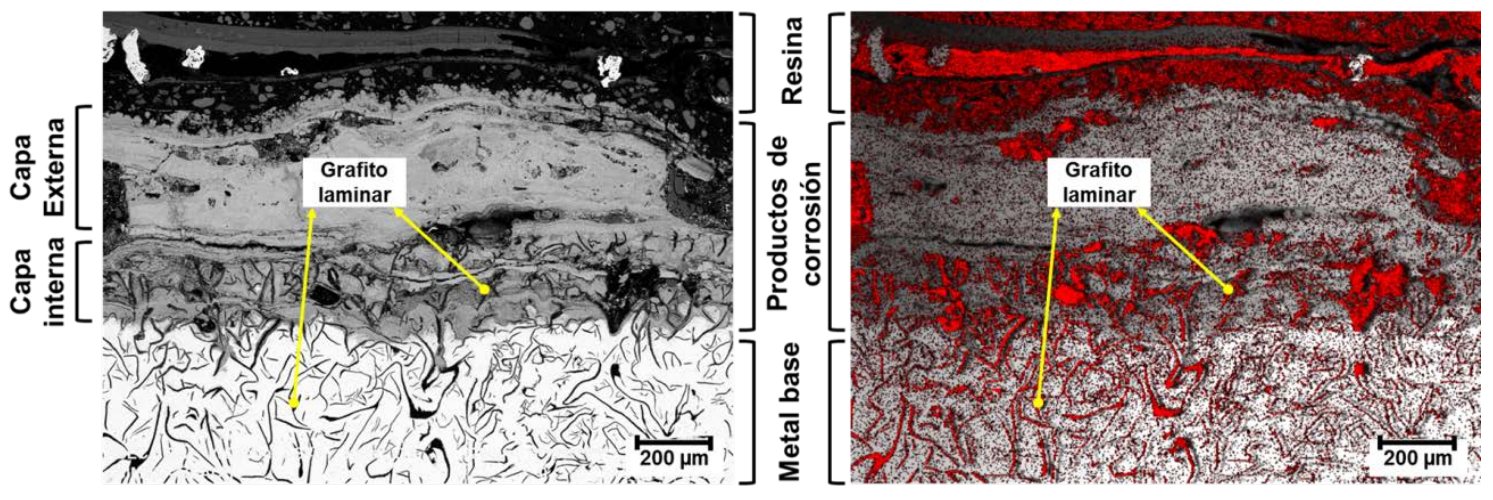

Figura 3: Imagen de microscopía electrónica de los productos de corrosión de la fundición gris, luego del ciclo 30.

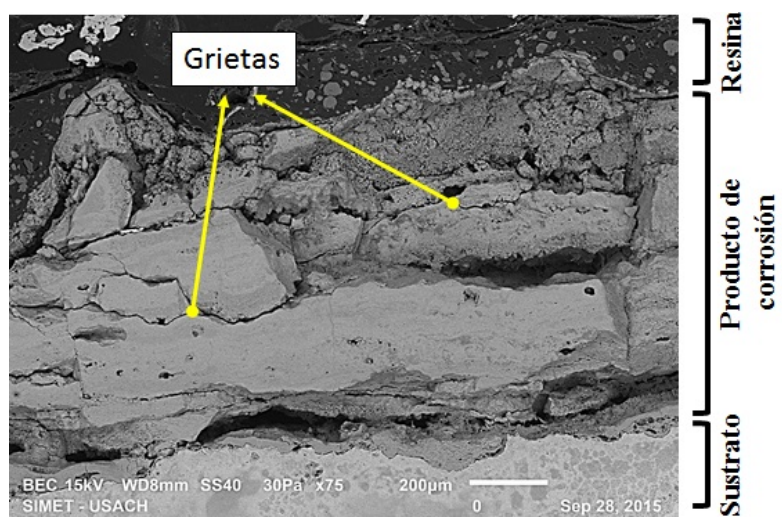

Figura 4: Imagen de microscopía electrónica de los productos de corrosión del acero estructural, luego del ciclo 30.

De acuerdo a las imágenes antes presentadas se observa que en la fundición se desarrolla un producto de corrosión con menos presencia de grietas, que las observadas para el acero estructural. Un producto de corrosión adherente y compacto y sin grietas protege al material, tal como lo describe Morcillo et al. [10], motivo por el que se esperarían menores tasas de corrosión en el tiempo para la fundición gris que para el acero estructural.

Además, en la Figura 3 se observa que el avance de la corrosión, en la fundición gris, se realiza a través de la matriz ferrítico-perlítica, dejando ancladas a su estructura las láminas de grafito de este material, lo que sería beneficioso, al crear sitios para la nucleación de FeOOH y con esto disminuir el tamaño de grano del producto de corrosión propiciando su adherencia y protección, tal como informa Kimura et al. [29] define para aceros autopatinables.

\subsection{Espesores corroídos}

La Figura 5, presenta los resultados de espesor corroído en el tiempo, luego de los ensayos de ciclado para la fundición gris y acero estructural estudiados (a la izquierda). En la misma imagen, a la derecha, se ilustran los resultados obtenidos por Southwell, pudiéndose inferir que la fundición gris presenta una mayor resistencia a la corrosión atmosférica, pues desarrolla menores espesores corroídos en el tiempo, siendo concordante con lo discutido con la sección previa, al comparar morfológicamente los productos de corrosión desarrollados.

En ambos casos se observa el cambio de pendiente luego del algún tiempo, respondiendo a un modelo del tipo bi-modal como el descrito por Melchers [23] o bien, bi-lineal como lo describen Albrecht y Hall [30]. 

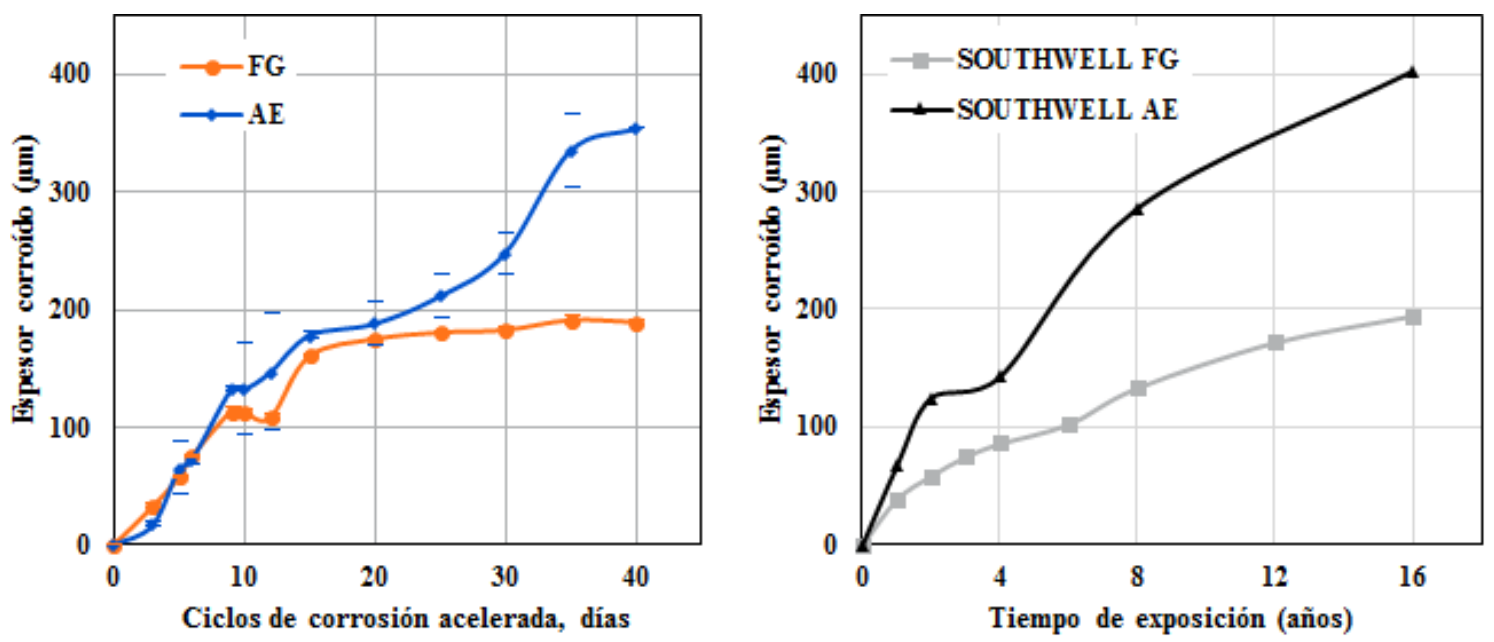

Figura 5: Espesores corroídos en el tiempo, a la izquierda ensayo de corrosión acelerada y a la derecha en tiempo real.

Dado que las composiciones para los aceros estructurales del estudio actual y el desarrollado por Southwell et al. [5], presentan similitudes, se ha homologado lo realizado por este equipo de trabajo para aceros autopatinables [4] y se han obtenido ajustes de corto y largo plazo para el acero estructural (Ecuaciones 3 y 4 y Figura 6). Luego, se ha determinado la equivalencia entre días de ensayo de corrosión atmosférica propuesto por este equipo y años de exposición a la intemperie según Southwell et al. [5]. A través de este procedimiento, se han construido gráficas comparativas entre los materiales estudiados por Southwell y los obtenidos a través de corrosión acelerada, véase Figura 7.

$C=78,55 t_{r}^{0,46}$,

$C=62,97\left(t_{r}-4,00\right)^{0,56}+148,97$,
$0 \leq t_{r} \leq 4$

$4 \leq t_{r} \leq 16$

C es el espesor corroído en $\mu \mathrm{m}$ y t $\mathrm{r}_{\mathrm{r}}$ el tiempo de exposición a la intemperie en años.

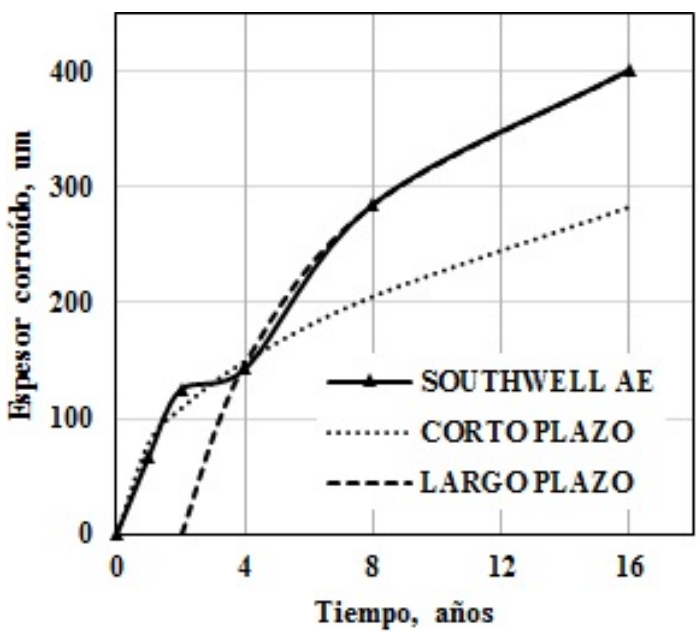

Figura 6: Ajustes de corto y largo plazo para AE Southwell. 

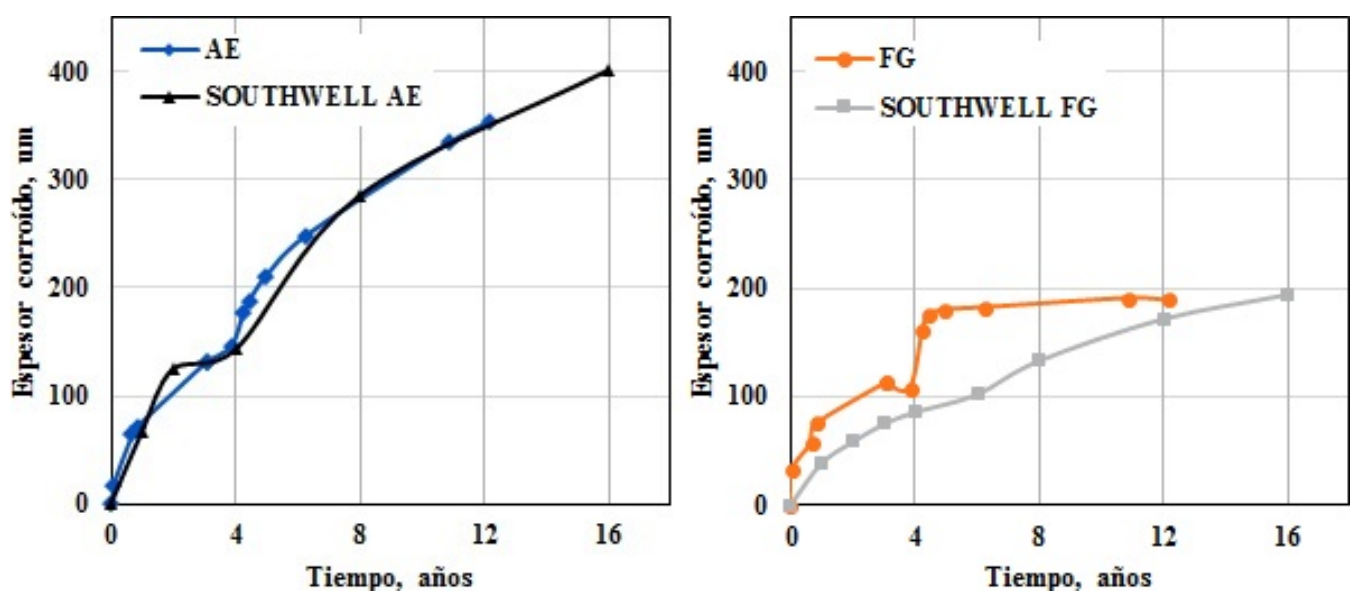

Figura 7: Comparación de resultados obtenidos por corrosión acelerada y exposición a la intemperie, en años.

Se observan diferencias en cuando a la resistencia a la corrosión atmosférica para el caso de las fundiciones grises, observando que la estudiada por Southwell et al. presenta menores espesores corroídos en el tiempo, siendo lo anterior justificado en la diferencia que experimentan en cuanto a sus composiciones químicas; se esperarían resultados similares al comparar resultados de fundiciones con similares contenidos de aleantes.

De acuerdo a los resultados presentados, se puede inferir que, tras 40 días (ciclos) de corrosión acelerada, con el ensayo propuesto, se han simulado poco más de 12 años de exposición a la intemperie en la zona costera del canal de Panamá, ajustándose los resultados de manera razonable para los aceros estructurales comparados por poseer similar composición química.

Se plantea como lineamiento futuro, la realización de ensayos de corrosión acelerada a materiales de composición concordante a los presentados por Southwell, con el propósito de validar aún más el método propuesto para la evaluación de resistencia a la corrosión atmosférica marina, a través de la obtención de similares resultados en pocas semanas.

En base a investigaciones anteriores [4, 14,15], donde se simularon exitosamente la corrosión atmoferica en las costas de Valparaíso, Chile, en el cual los productos de corrosión eran similares a los de largo plazo reportados por la literatura [25, 29, 31], y al aplicar la misma metodología, pero con los valores de exposición real reportados en al canal de panamá por Melcher [6], se puede establecer una buena relación entre el ambiente simulado y real, incluso manifestando el comportamiento bimodal.

\section{CONCLUSIONES}

Mediante un ensayo de corrosión atmosférica marina simulada, se puede predecir su comportamiento en tiempo real de exposición, incluso reproduciendo el comportamiento bimodal de aleaciones ferrosas. A través de esta metodología se puede estudiar el comportamiento a la corrosión atmosférica marina de nuevas aleaciones, pudiendo evaluar el comportamiento de elementos aleantes como el cobre, níquel o titanio en pos de una mejora del comportamiento a la corrosión atmosférica marina de los aceros y fundiciones.

\section{AGRADECIMIENTOS}

Los autores desean agradecer al proyecto DICYT, Código 051614AA, Vicerrectoría de Investigación, Desarrollo e Innovación de la Universidad de Santiago de Chile y al laboratorio SIMET-USACH por el apoyo prestado al presente trabajo.

\section{BIBLIOGRAFÍA}

[1] MODERN CASTING STAFF, “49th Census of world casting production”, Modern Casting, v. 103, n. 12, pp. 26-31, Dic. 1996.

[2] ASTM Standard A48/A48M - 03 (Reapproved 2012), 2012, “ Standard Specification for Gray Iron Castings”, ASTM International, West Conshohocken, PA, 10.1520/A0048_A0048M-03R12, http://www.astm.org. [3] ASTM Standard A36/A36M-14, 2014, "Standard Specification for Carbon Structural Steel”, ASTM In- 
ternational, West Conshohocken, PA, DOI: 10.1520/A0036_A0036M-14, http://www.astm.org.

[4] ARTIGAS, A., MONSALVE, A., SIPOS, K., et al., "Development of accelerated wet-dry cycle corrosion test in marine environment for weathering steels", Corrosion Engineering, Science and Technology", v. 50, n. 8, pp. 628-632, Feb. 2015.

[5] SOUTHWELL, C., BULTMAN, J., ALEXANDER, A., "The corrosion rates of structural metals in seawater, fresh water and tropical atmospheres: Summary of a sixteen-year exposure study", Modern Casting, v. 19, n. 3, pp. 179-183, Mar. 1969.

[6] MELCHERS, R., "Long-term corrosion of cast irons and steel in marine and atmospheric environments", Corrosion Science, v. 68, n. 1, pp. 186-194, Mar. 2013.

[7] MELCHERS, R., JEFFREY, R. "The critical involvement of anaerobic bacterial activity in modelling the corrosion behaviour of mild steel in marine environments”, Electrochimica Acta, v. 54, n. 1, pp. 80-85, Mar. 2008.

[8] MELCHERS, R., "Microbiological and abiotic processes in modelling longer-term marine corrosion of steel”, Bioelectrochemistry, v. 97, n. 1, pp. 186-194, Jun. 2014.

[9] VERA, R., DELGADO, D., ARAYA, R., et al., "Construcción de mapas de corrosión atmosférica de chile: Resultados preliminares”, Revista Latinoamericana de Metalurgia y Materiales, v. 32, n. 2, pp. 269276, Nov. 2012.

[10] MORCILLO, M., CHICO, B., DÍAZ, I., et al., "Atmospheric corrosion data of weathering steels. A review”, Corrosion Science, v. 77, n.1, pp. 6-24, Sept. 2013.

[11] MORCILLO, M., CHICO, B., DÍAZ, I., et al., "Atmospheric corrosion of Ni-advanced weathering steels in marine atmospheres of moderate salinity”, Corrosion Science, v. 76, n. 1, pp. 348-360, Jul. 2013.

[12] SURNAM, B., CHUI, C., XIAO, H., et al., "Investigating atmospheric corrosion behavior of carbon steel in coastal regions of Mauritius using Raman Spectroscopy”, Revista Matéria, v. 21, n. 1, pp. 157-168, Mar. 2016.

[13] OLAWALE, J., ODUSOTE, J., RABIU, A., et al., "Evaluation of Corrosion Behaviour of Grey Cast Iron and Low Alloy Steel in Cocoa Liquor and Well Water”, Journal of Minerals and Materials Characterization and Engineering, v. 1, n. 2, pp. 44-48, Mar. 2013.

[14] ARTIGAS, A., BUSTOS, O., GARZA-MONTES-DE-OCA, N., et al., "Influencia de Ni y Ti en la respuesta a la corrosión marina simulada de un acero autopatinable A242”, Revista Matéria, v. 20, n. 3, pp. 646652. Sept. 2015.

[15] ARTIGAS, A., BUSTOS, O., GARZA-MONTES-DE-OCA, N., et al., "Comportamiento a la corrosión atmosférica marina de aceros autopatinables con estructura ferrítico perlítica y ferrítico martensítica”, Revista Matéria, v. 20, n. 3, pp. 659-667, Sep. 2015.

[16] WANG, J., WANG, Z., “Corrosion behaviour of weathering steel in diluted Qinghai salt lake water in a laboratory accelerated test that involved cyclic wet/dry conditions", Materials Chemistry and Physics, v. 124, n- 2-3, pp. 952-958, Dic. 2010.

[17] DUNN, D., BROSSIA, C., BOGART, M., et al., Corrosion of Iron Under Alternating Wet and Dry Conditions, Corrosion 99, NACE-99223, San Antonio, Texas, USA, 25-30 Abril 1999.

[18] WANG, Z., YIN, F., WU, L., SUN, Y., ZHANG, W., "Corrosion Resistance on High Strength Bainitic Steel and 09cupcrni after Wet-Dry Cyclic Conditions", Journal of Iron and Steel Research, v. 20, n. 2, pp. 72-78, Feb. 2013.

[19] ZHANG, W., YANG, S., GUO, J., et al., "Incubation and development of corrosion in micro-estructures of low alloys steels under a thin liquid film of $\mathrm{NaCl}$ aqueous solution", International Journal of Minerals, Metallurgy, and Materials, v.17, n. 6, pp. 748-755, Dic. 2010.

[20] DONG, J., HAO, L., ZHANG, S., et al., "A study the evolution of rust on Mo-Cu-bearing fire-resistant steel submitted to simulated atmospheric corrosion”, Corrosion Science, v. 54, n. 1, pp. 244-250, Ene. 2012.

[21] HAO, L., ZHANG, S., DONG, J., et al., "Evolution of atmospheric corrosion of MnCuP weathering steel in a simulated coastal-industrial atmosphere”, Corrosion Science, v. 59, n. 1, pp. 270-276, Mar 2012.

[22] AKIYAMA, E., LI, S., SHINOHARA, T., et al., "Hydrogen entry into Fe high strength steels under simulated atmospheric corrosion”, Electrochemical Acta., v. 56, n. 4, pp. 1799-1805, Ene. 2011.

[23] MELCHERS, R.E. Modeling of Marine Corrosion of Steel Specimens, Corrosion Testing in Natural Waters, ASTM STP 1300, Philadelphia, Pensilvania, USA, 1 Abril 1997. 
[24] ASTM Standard B117 - 16, 2016, "Standard Practice for Operating Salt Spray (Fog) Apparatus", ASTM International, West Conshohocken, PA, DOI: 10.1520/B0117-16, http://www.astm.org.

[25] KIMURA, T., NASU, S., TAZAKI, T., et al., "Mossbauer spectroscopic study of rust formed on a weathering steel and a mild steel exposed for a long term in an industrial environment", Materials Transactions, v. 43, pp. 694-703, Feb. 2002.

[26] BADAN, B., MAGRINI, M., RAMOUS, E., "A study of the microbiological-corrosion products of steel and cast iron pipes in fresh water", Journal of Materials Science, v. 27, n. 1, pp. 1951-1954, Ene. 1991.

[27] MOHEBBI, H., LI, C. Q., “Experimental Investigation on Corrosion of Cast Iron Pipes”, International Journal of Corrosion, v. 1, n. 1, Mar. 2011.

[28] ASTM Standard G1 - 03 (Reapproved 2011), 2012, " Standard Practice for Preparing, Cleaning, and Evaluating Corrosion Test Specimens", ASTM International, West Conshohocken, PA, DOI: 10.1520/G0001-03R11, http://www.astm.org.

[29] KIMURA, M., KIHIRA, H., OHTA N., et al., "Control of $\mathrm{Fe}(\mathrm{O}, \mathrm{OH})_{6}$ nano-network structures of rust for high atmospheric-corrosion resistance”, Corrosion Science., v. 47, n. 10, pp. 2499-2509, Jun. 2005.

[30] ALBRECHT, P., HALL, T., JR., "Atmospheric Corrosion Resistance of Structural Steels”, Journal of Materials in Civil Engineering., v. 12, n. 1, pp. 2-24, Feb. 2003.

[31] OKADA H., HOSOI Y., YUKAWA K., et al., "Structure of the protective and decorative rust formed on low-alloy steels in the atmosphere”, Trans. ASM, v. 62, pp. 278-281. 1969 